\title{
Investigation of psoriasis skin tissue by label-free multi-modal imaging: a case study on a phototherapy-treated patient
}

This article was published in the following Dove Press journal:

Psoriasis: Targets and Therapy

\author{
Nisha Rani Agarwal ${ }^{1, *}$ \\ Masoumeh Dowlatshahi \\ Pour $^{2, *}$ \\ Maria Siekkeri Vandikas ${ }^{3}$ \\ Noora Neittaanmäki ${ }^{4}$ \\ Amra Osmancevic ${ }^{3}$ \\ Per Malmberg ${ }^{2}$ \\ 'Department of Biology and Biological \\ Engineering, Chalmers University of \\ Technology, Gothenburg, Sweden; \\ ${ }^{2}$ Department of Chemistry and Chemical \\ Engineering, Chalmers University of \\ Technology, Gothenburg, Sweden; \\ ${ }^{3}$ Department of Dermatology, \\ Sahlgrenska University Hospital at \\ Sahlgrenska Academy, University of \\ Gothenburg, Gothenburg, Sweden; \\ ${ }^{4}$ Department of Clinical Pathology, \\ Institutes of Biomedicine and Clinical \\ Sciences, Sahlgrenska Academy, \\ University of Gothenburg, Gothenburg, \\ Sweden
}

*These authors contributed equally to this work

\begin{abstract}
Background: Psoriasis is a systemic inflammatory disease characterized by epidermal proliferation in the skin. Altered lipid metabolism is considered to be a central factor in the psoriatic etiopathogenesis. Thus, it is necessary to visualize chemical specificity of the samples for better medical diagnosis and treatment. Here, we investigate its role in the development of psoriatic lesions, before and after ultraviolet phototherapy, in a case study.

Methods: The distribution and morphology of different lipids and fibrous proteins in psoriatic (lesional) tissues were visualized by two complementary label-free imaging techniques: 1) non-linear microscopy (NLM), providing images of lipids/proteins throughout the skin layers at submicrometer resolution; and 2) mass spectrometry imaging (MSI), offering high chemical specificity and hence the detection of different lipid species in the epidermal and dermal regions. A conventional method of histological evaluation was performed on the tissues, with no direct comparison with NLM and MSI.
\end{abstract}

Results: Psoriatic tissues had a higher lipid content, mainly in cholesterol, in both the epidermal and dermal regions, compared to healthy tissues. Moreover, the collagen and elastin fibers in the psoriatic tissues had a tendency to assemble as larger bundles, while healthy tissues showed smaller fibers more homogeneously spread. Although phototherapy significantly reduced the cholesterol content, it also increased the amounts of collagen in both lesional and non-lesional tissues.

Conclusion: This study introduces NLM and MSI as two complementary techniques which are chemical specific and can be used to assess and visualize the distribution of lipids, collagen, and elastin in a non-invasive and label-free manner.

Keywords: psoriasis skin tissue, non-linear microscopy, lipids, collagen, time-of-flight secondary ion mass spectrometry (ToF-SIMS)

\section{Introduction}

Psoriasis is an inflammatory immune-mediated disease affecting primarily the skin and potentially the joints and other tissues. ${ }^{1,2}$ The prevalence of psoriasis is estimated as $120-180$ million people worldwide. ${ }^{3}$ Plaque psoriasis is clinically presented by thick, scaly, and sometimes itching lesions distributed on the scalp, trunk, and extensor sides of the limbs. Mild to moderate disease is usually treated with narrowband ultraviolet B therapy (NBUVB), ${ }^{4,5}$ and severe psoriasis is treated with systemic therapies including biological drugs. ${ }^{6}$ Ultraviolet (UV) rays have an important role to play in the healing of skin disorders and hence are a very common treatment for varied skin disorders. ${ }^{7}$
Correspondence: Nisha Rani Agarwal Faculty of Science, University of Ontario Institute of Technology, 2000 Simcoe Street North, Oshawa, LIG 0C5, ON Canada

Email nisha.agarwal@uoit.ca 
However, in the past few decades it has become clear that psoriatic skin lesions are just one of many symptoms of this systemic disease, which is also associated with increased risks of cardiovascular disease, hypertension, atherosclerosis, diabetes mellitus type 2, and obesity, as reviewed by Tobin et al. ${ }^{8}$ Dysfunctional lipid metabolism is proposed as one of the central underlying mechanisms, resulting in altered ceramide/phospholipid/cholesterol contents $^{9-11}$ and lipid structures ${ }^{12}$ in the epidermis, as well as abnormal cholesterol levels in the lipoprotein fractions in blood plasma. ${ }^{9,13,14}$ There is evidence that all these changes are caused by altered cholesterol efflux capacity. ${ }^{12}$ To understand the role of and target the altered lipid metabolism in psoriatic skin lesions, there is a great need for new technologies allowing the visualization of the distribution of lipids throughout the skin layers at high spatial resolution and with high chemical specificity.

The diagnosis of psoriasis is often clinical. Both the diagnosis and the effect of treatment in psoriasis are highly reliant on inspection of scaly patches on the skin and dermoscopy and histopathological images, which limits doctors to information that has changed at the chemical level. ${ }^{15}$ Clinicians use standard biopsied tissues with H\&E staining for identifying specific psoriatic markers, but are limited to bright-field images and single-photon microscopy. ${ }^{16}$ This routine procedure does not allow detection of changes at the lipid level.

In this study, we introduce a combination of non-linear microscopy (NLM) ${ }^{17,18}$ and mass spectrometry imaging $(\mathrm{MSI})^{19,20}$ to map distributions of different lipid species with high spatial resolution, along with changes in the morphology and contents in fibrous skin matrix proteins. The lipid-associated psoriasis markers can be established with high sensitivity and specificity, ${ }^{15}$ and be associated to the treatment strategy and dosage. This is the first time that these two label-free techniques have been combined to obtain high-quality imaging and semi-quantitative molecular information from psoriasis tissue, and our findings indicate the applicability of this technique for future studies.

NLM is based on the non-linear processes that take place in a material by excitation with multiple photons, providing various advantages. ${ }^{21-24}$ The coherent anti-Stokes Raman spectroscopy (CARS), and two-photon excitation fluorescence (TPEF) and second-harmonic generation (SHG) microscopy techniques provide complementary information on the molecular composition and organization of skin. ${ }^{17}$ They map entities rich in carbon-hydrogen $(\mathrm{C}-\mathrm{H})$ bonds such as lipids and proteins (CARS), ${ }^{25,26}$ inherent fluorophores present in the skin such as keratin, ${ }^{27}$ NADH and elastin $^{28}$ (TPEF), ${ }^{29}$ and fibrous proteins such as collagen (SHG). ${ }^{30,31} \mathrm{MSI}$ and especially time-of-flight secondary ion mass spectrometry (ToF-SIMS), one of the most sensitive analytical techniques in surface science, has become a powerful technique for determining, mapping, and visualizing the distribution of endogenous biological molecules. ${ }^{32,33}$ ToF-SIMS is an ultra-high-vacuum technique in which a high-energy and focused ion beam is used to bombard, ionize, and analyze the surface of a solid sample, providing information about chemicals and the composition of the analyzed surface. The technique is well suited to the detection of elements, lipids, and metabolites ${ }^{20,34}$ with mass to charge ratios up to $m / z 1,000-1,500$ and a lateral resolution from $200 \mathrm{~nm}$ to $1-2 \mu \mathrm{m} .{ }^{35}$ The samples in ToF-SIMS-based MSI can be directly used for analysis and the technique requires no pretreatment, such as labeling with probes or coating with matrix, which makes it particularly efficient for non-targeted analysis of tissue sections, ${ }^{36-39}$ and for a multi-modal approach with NLM.

There have been several studies on different multimodal approaches for the study of skin and other biological systems. ${ }^{16,40-42}$ We conduct a study on the combination of NLM and MSI, illustrating its strength for the visualization of lipids and structural matrix proteins, where NLM provides high spatial resolution in three dimensions and MSI provides high chemical specificity for the identification of different lipid species. Moreover, we are interested in comparing the information acquired from NLM and MSI with findings on routine histological evaluation. In particular, this study brings forward new perspectives by highlighting various aspects in the fast and effective analysis of psoriatic tissue, which include: 1) performing a case study on a psoriatic lesion before and after treatment with NBUVB radiation in comparison with healthy tissue from the same patient; 2) examining the tissue samples via multi-modal techniques, namely NLM and MSI; 3) combining qualitative information from CARS, SHG, and TPEF with quantitative information and chemical specificity produced by ToF-SIMS; and 4) characterizing the different layers of the skin. The highlights of the two techniques are that NLM provides information on the overall chemical composition of the skin while ToF-SIMS demonstrates the chemical specificity at the molecular level. In this report, we present evidence on the effectiveness of the two complementary techniques to be used for characterization of lipid contents and structural proteins in psoriatic tissues. This 
pioneering knowledge can increase our insight into the pathophysiology of psoriasis and its relationship to altered lipid metabolism. ${ }^{43}$

Through this study, we are able to demonstrate the characterization of psoriatic, lesional tissues along with healthy tissues before and after NBUVB treatment using two techniques, NLM and MSI. The techniques complement each other well, with one giving visual structural details of different chemical components in the skin and the other exemplifying the molecular composition of the different chemical components in the skin tissue, with lipid content and collagen structure as specific markers. Our aim is to introduce these two techniques to dermatologists as easy and time-saving methodologies providing detailed results for diagnostic and treatment considerations in psoriasis. Furthermore, this pioneering knowledge can increase insight into the pathophysiology of psoriasis and its correlated conditions, including disturbances in lipid metabolism. $^{43}$

\section{Materials and methods}

\section{Patient details and sample preparation}

A female patient aged 60 years (with skin type III according to Fitzpatrick), ${ }^{44}$ with moderate chronic plaque psoriasis (Psoriasis Area and Severity Index [PASI] =7.7), came to the dermatological clinic to receive phototherapy. The study was approved by the Ethics Committee at the University of Gothenburg and the National Data Inspection Board (approval number DNR:089-12, T285-16). The patient's written informed consent was obtained for case details and publishing accompanying images. The patient was treated with NBUVB therapy (311 nm, according to standard protocol, 28 sessions, total dose $3.0 \mathrm{~J} / \mathrm{cm}^{2}$ (increasing from 0.3 to $3.0 \mathrm{~J} / \mathrm{cm}^{2}$ ) and psoriasis significantly improved (PASI $=0.6$ ). Four 4 $\mathrm{mm}$ punch biopsies were taken, one from intralesional psoriatic skin and one from perilesional healthy skin (about $2 \mathrm{~cm}$ from the psoriasis plaque) before and 24 hours after the last NBUVB session (Table S1). The biopsies were wrapped in aluminum foil and frozen at $-70^{\circ} \mathrm{C}$. Each sample was sectioned into $10 \mu \mathrm{m}$ thin slices and placed on conductive glass slides. The whole process is illustrated in Figure S1. The sections on the slides were used as they were for NLM and ToF-SIMS measurements; no treatment was done before the measurements. For histological assessment, the frozen sectioned slides were stained with H\&E (Figure S2).

\section{NLM and analysis}

NLM was conducted using the $532 \mathrm{~nm}$ (frequency doubled) beam of the $\mathrm{Nd} \mathrm{YVO}_{4}$ pump laser (picoTRAIN; HighQ Laser, Santa Clara, CA, USA) to pump the Optical Parametric Oscillator (OPO) (Levante Emerald OPO; APE, Berlin, Germany) to provide an output of $817 \mathrm{~nm}$. The pump beam has a pulse length of $7 \mathrm{ps}$ and repetition rate of $76 \mathrm{MHz}$. The $817 \mathrm{~nm}$ beam is coupled into the scanning head unit (Nikon C1) of the Nikon Eclipse TE2000-E microscope. The beam was focused using a high NA oil objective (Nikon Plan Fluor $40 \times / 1.30$ ). The CARS signal was collected in transmission with a high NA lens into a single-photon-counting photomultiplier tube (HPM-100-40; Becker \& Hickl, Berlin, Germany). The SHG and TPEF signals emitting in the blue and green region of the visible spectrum were collected simultaneously in the epi-direction (epi meaning backword or in reflection, in our case) with two other single-photon counters (HPM-100-50; Becker \& Hickl). The SHG and TPEF epi-collection was separated by a DiO2-R442 dichroic mirror. The laser power impinging on the sample was around $6 \mathrm{~mW}$ and $7 \mathrm{~mW}$ for the pump and Stokes beams, respectively. The samples were characterized by NLM with simultaneous CARS, SHG, and TPEF imaging at emissions of $663 \mathrm{~nm}, 405 \mathrm{~nm}$, and 514 $\mathrm{nm}$, respectively. CARS, SHG, and TPEF signals were collected with band-pass filters 661/20, 405/10 and 514/ 30 respectively. Z-stacks were acquired for each sample, with $0.5 \mu \mathrm{m}$ step size for the $10 \mu \mathrm{m}$ sample thickness. Each single acquisition in the Z-stack was taken in 53 seconds with a resolution of 512 pixels for a $168.4 \mu \mathrm{m}$ field of view. Analysis was performed on two regions per two samples. The images were processed in ImageJ software. The outline of the set-up is shown in Figure S3.

\section{ToF-SIMS and analysis}

All samples were analyzed using a TOF.SIMS 5 instrument (ION-TOF, Münster, Germany) equipped with a Bi cluster ion gun. Data were acquired in the high-current bunch mode (mass resolution $\mathrm{m} / \Delta \mathrm{m}=\sim 5000$ ), ${ }^{45}$ with a pulsed primary ion current of $0.30 \mathrm{pA}$ at $25 \mathrm{keV}$. The ion dose density of $\mathrm{Bi}^{3+}$ was kept at $<2 \times 10^{11}$ ions $/ \mathrm{cm}^{2}$ and therefore below that of the static limit, ie, $<10^{13}$ ions $/ \mathrm{cm}^{2}$, to minimize surface damage. Low-energy electrons were used for charge compensation. All ToFSIMS spectra and images were acquired and processed using the Surface Lab software (version 6.3; ION-ToF, 
Münster, Germany). The spectra were then internally calibrated to signals of common fragment peaks at $[\mathrm{C}]^{+},[\mathrm{CH}]^{+},\left[\mathrm{CH}_{2}\right]^{+}$, and $\left[\mathrm{CH}_{3}\right]^{+}$.

\section{Statistics}

To assess changes in collagen and lipid species among the samples from the patient, three specific region of interest (ROIs) on both epidermis and dermis were separately created and semi-quantitatively evaluated using Welch's $t$-test. Welch's $t$-test is often used to assess MSI data. ${ }^{46,47}$ This statistic is robust for testing means unless the distribution is non-normal or the size of the groups is very different. ${ }^{48}$

\section{Results}

\section{Label-free imaging of psoriatic tissue}

The collagen content, as well as some specific lipid species such as phosphatidylcholine, cholesterol, and diacylglycerols (DAGs), were mapped in the skin tissue sections from control lesions and psoriasis lesions before and after NBUVB treatment, using NLM and ToF-SIMS imaging. For ToF-SIMS, by reference to previously reported literature, ${ }^{33}$ the following mass peaks were assigned as characteristic positive fragment ions corresponding to collagen: $m / z 30\left(\mathrm{CH}_{4} \mathrm{~N}^{+}\right), m / z 43\left(\mathrm{CH}_{3} \mathrm{~N}_{2}{ }^{+}\right), m / z 44\left(\mathrm{C}_{2} \mathrm{H}_{6} \mathrm{~N}^{+}\right)$, and $m / z 81\left(\mathrm{C}_{4} \mathrm{H}_{5} \mathrm{~N}_{2}{ }^{+}\right)$. The mass peaks corresponding to $m / z 184$ (phosphatidylcholine head group $\left[\mathrm{C}_{5} \mathrm{H}_{15} \mathrm{PO}_{4} \mathrm{~N}\right]^{+}$) and $m / z 369$ (cholesterol $\left[\mathrm{M}^{+} \mathrm{H}^{-} \mathrm{H}_{2} \mathrm{O}\right]^{+}$), as well as DAG lipid species, were also assigned. Part of a ToF-SIMS mass spectrum from a tissue section including $\mathrm{m} / \mathrm{z} 30, \mathrm{~m} / \mathrm{z} 43, \mathrm{~m} / \mathrm{z}$ 44, $m / z 81, m / z 184$, and $m / z 369$, as well as DAG-related lipid peaks, is illustrated in Figure S4 (A-D).

The advantages of optical NLM are submicrometer spatial resolution, chemical specificity allowing separation of major categories of biomolecules (lipids vs proteins), labelfree detection, and minimum photo-damaging (use of nearinfrared [NIR] laser light). In CARS microscopy, an enhanced signal is generated when two photons with a slight energy (ie, wavelength) difference interact with a molecule by matching the energy difference to that of a resonant inherent molecular vibration, further probed by a third photon. Here, we map lipids by probing the $2850 \mathrm{~cm}^{-1}$ vibration characteristic of the $\mathrm{C}-\mathrm{H}$ stretch vibration of the lipid acyl chain. ${ }^{25}$ Furthermore, TPEF signals in the blue/ visible region are generated from inherent fluorophores present in the skin upon the absorption of two NIR photons. Here, elastin, NADH, and keratin are the most important contributors in skin. ${ }^{27,28}$ Lastly, the SHG signal is produced when two NIR photons interact with the target molecule and generate higher order vibrations at double the frequency. They sum up constructively for non-centrosymmetrical molecules such as collagen. ${ }^{49}$ Since the epidermal stratum corneum consists of anucleate, flattened cells filled with keratin, being embedded in lipids, cholesterol, free saturated fatty acids and ceramides, ${ }^{50}$ it shows up well in both the CARS and the TPEF images, though representing the distribution of lipids and keratin, respectively. Because of the intracellular contents of lipid-rich lamellar bodies, ${ }^{51}$ and keratin in the keratinocytes below the stratum corneum, they can also be distinguished based on their molecular contents in both the CARS and TPEF images. In the dermal layer, lipids in the sebaceous glands (CARS), elastin/NADH (TPEF), and collagen (SHG) show up on the corresponding images. However, as a result of refractive index mismatch at the interfaces of the collagen fibers, they appear in the CARS images as shadowing artifacts. ${ }^{52}$ This can be compensated for by subtracting the non-resonant background, influenced by the same phenomenon.

\section{Histopathological analysis}

Histological evaluation of the lesional sample 1 (before treatment) represents typical findings of psoriasis. There is a confluent parakeratotic surface, psoriasiform elongated rete ridges, missing granular cells layer, and perivascular lymphohistiocytic inflammation (Figure S2). Histologically, the lesional sample 2 (after treatment) represents chronic dermatitis, ie, it does not fulfill the criteria of psoriasis but is not completely histologically healed. There is irregular acanthotic epidermis with narrow long rete ridges. There is also focal basal pigment, probably due to the phototherapy. No parakeratosis is visible. A layer of granular cells is visible. There is mild perivascular lymphohistiocytic inflammation (Figure S2).

Sample 3 (healthy skin before treatment) could not be analyzed using histopathology owing to artifacts during the processing of the sample. Nevertheless, sample 3 could be easily characterized using the multi-modal microscopy approach. Healthy sample 4 (after treatment) showed histological findings of normal skin without signs of inflammation or psoriasis. In this biopsy sample, light basal pigmentation is also visible, probably due to the phototherapy (Figure S2).

\section{Distribution of collagen and lipid species in epidermis}

The epidermis region is the foremost layer, consisting of the epithelium (red) and basal lamina (purple), as indicated 
in the bright-field images of Figure 1. Our first observation relates to the size of the epithelium layer of the epidermis. The thickness of the epithelium region is around $60 \mu \mathrm{m}$ for the lesional samples 1 (before treatment) and 2 (after treatment) and 15-20 $\mu \mathrm{m}$ for the healthy samples 3 (before treatment) and 4 (after treatment). This indicates that the epithelium in the case of psoriasis tissue increases approximately three- to four-fold. This thickening of the epidermis can be detected in routine histology. In addition, after NBUVB treatment for both samples 2 and 4, there is not much change in the epithelium size from their respective skin conditions, either psoriatic or healthy. Unlike the processing of histological sample 3, NLM and MSI measurements and analysis were unaffected by the sample preparation methodology. From the first channel CARS (red) of sample 1 (lesional tissue before treatment), a layer of tissue is observed just below the epithelium signifying a high concentration of $\mathrm{C}-\mathrm{H}$ bonds, as shown by an arrow in the CARS channel of row 1, which is absent in samples 2 (lesional tissue after treatment), 3 (healthy tissue before treatment), and 4 (healthy tissue after treatment). This accumulation of $\mathrm{C}-\mathrm{H}$ bonds in the psoriatic tissue appears to be mainly lipids, since ToF-SIMS could detect a clear enrichment of cholesterol $(\mathrm{m} / \mathrm{z} 369)$ in the epidermis area. This cholesterol-rich region appeared to be immediately affected by NBUVB treatment since it did not appear in the healthy or NBUVB-treated tissues. From the second channel SHG (green), no collagen is observed in the basal lamina region of the epidermis for any of the sample conditions, except in the bulky mass below the

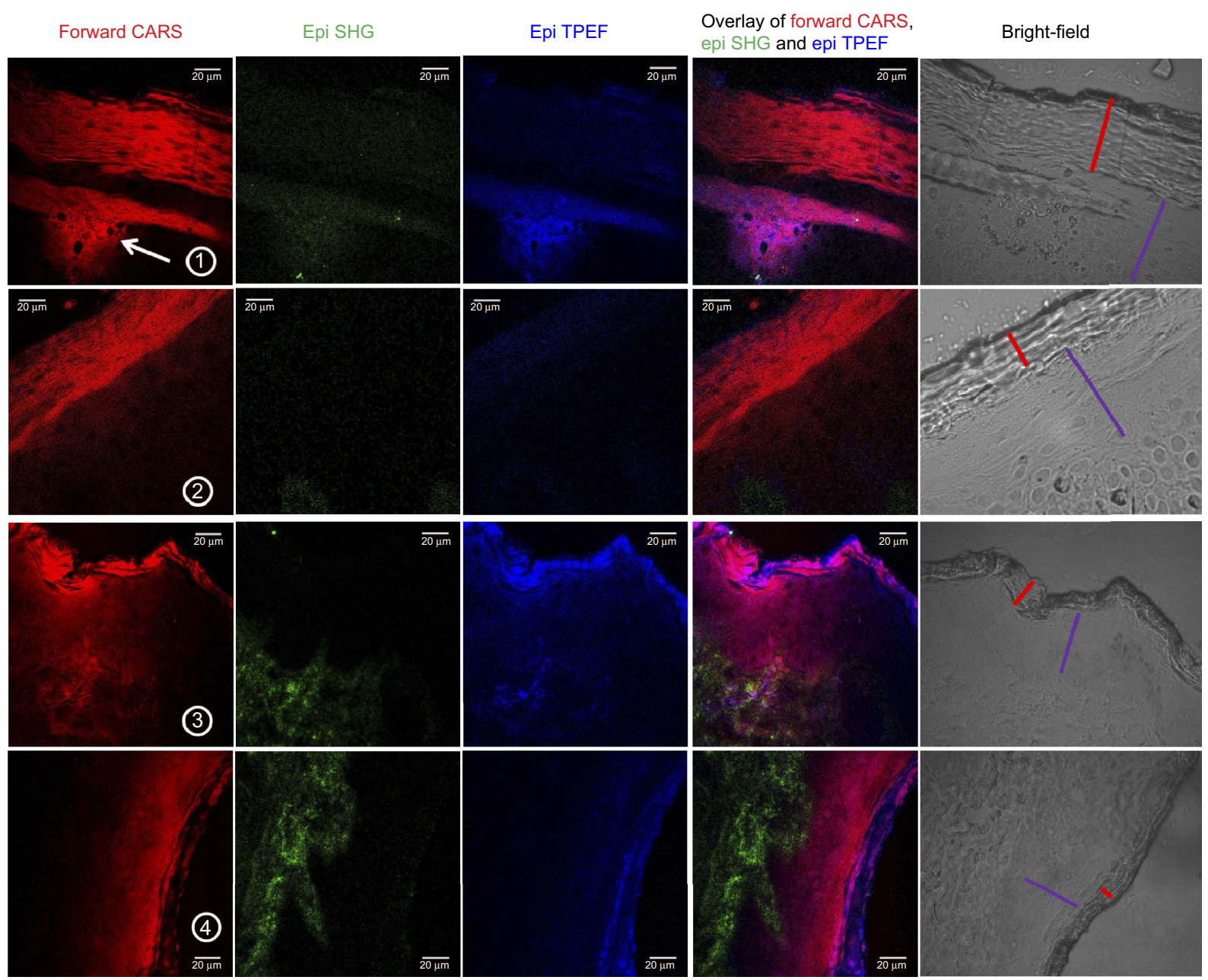

Figure I Non-linear microscopy of the epidermal region of skin tissue for healthy and treated biopsies: display of forward CARS, epi SHG, and epi TPEF, overlay of CARS, SHG, and TPEF, and bright-field images in the epidermis region of the skin for each sample condition. Row I: lesional psoriasis tissue before treatment; row 2: psoriasis tissue treated with NBUVB; row 3: healthy tissue; and row 4: healthy tissue treated with NBUVB. The bright-field images indicate the epithelium (red line) and basal lamina (purple line). Scale bar: $20 \mu \mathrm{m}$.

Abbreviations: CARS, coherent anti-Stokes Raman spectroscopy; NBUVB, narrowband ultraviolet B; SHG, second-harmonic generation microscopy; TPEF, two-photon excitation fluorescence microscopy. 
epithelium in the lesional sample 1. Moreover, the TPEF channel shows a higher presence of elastin and NADH in the epithelium of the healthy tissue in samples 3 and 4 and treated psoriatic tissue of sample 2 than in the lesional sample 1 . It is mostly present in the bulky layer below the epithelium in the lesional sample 1.

The ToF-SIMS technique was used to statistically elucidate the changes in signal intensity of collagen and lipid species among the samples. The average values of these peaks, which were collected from three specific ROIs on the epidermis region of each skin section, are shown in Figure 2 ( $p \leq 0.05$, significant values marked with asterisks). The effect of psoriasis on collagen, as well as phosphatidylcholine, cholesterol, and DAG lipid species, in the epidermis region of the skin is shown in Figure 2(A), where lesional tissue before treatment (sample 1) was compared with healthy tissue before treatment (sample 3 ). There average intensities of all collagen-related peaks
( $m / z 30,43,44$, and 81) appear significantly higher in the healthy sample, whereas cholesterol $(\mathrm{m} / z$ 369) is significantly decreased compared to the lesional skin. ${ }^{54,55}$ No significant changes are observed for phosphatidylcholine ( $m / z$ 184) between lesional and healthy samples 1 and 3 before NBUVB treatment. To see the effect of NBUVB treatment on psoriasis, the average intensities for the lesional samples 1 and 2, before and after treatment, respectively, were compared, as shown in Figure 2(B). It is evident that in sample 2, two collagen-related peaks of $\mathrm{m} / \mathrm{z} 30$ and 43 are substantially increased, but cholesterol ( $m / z$ 369) is significantly reduced. Comparison of the healthy samples 3 and 4, before and after NBUVB treatment, is shown in Figure 2(C), where a considerable increase in collagen $(\mathrm{m} / \mathrm{z} 30,43$, and 44$)$ as well as phosphatidylcholine $(\mathrm{m} / \mathrm{z} 184)$ can be seen, whereas cholesterol $(\mathrm{m} / \mathrm{z} 369)$ and also some DAG species $(\mathrm{m} / \mathrm{z} 519$, 521, and 523) are significantly decreased after treatment.

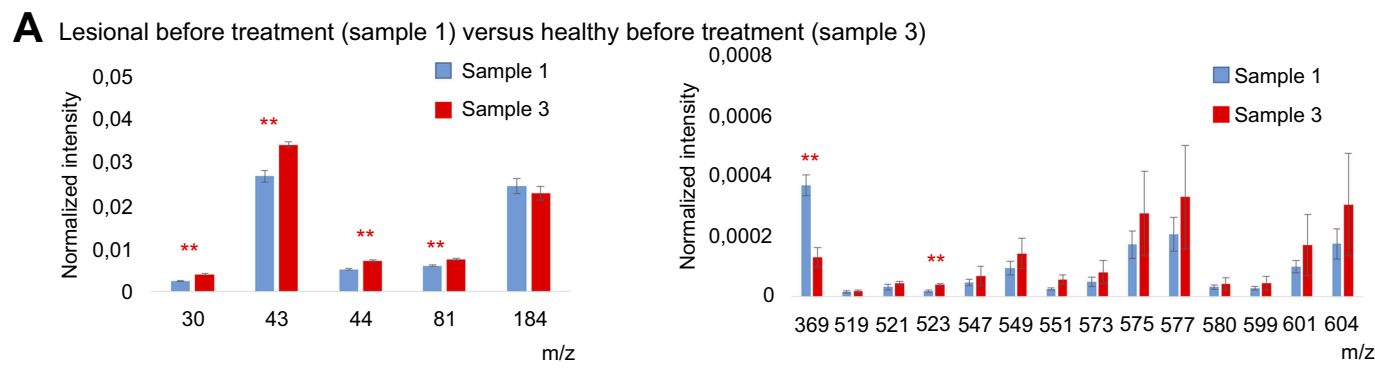

B Lesional before treatment (sample 1) versus lesional after treatment (sample 2)
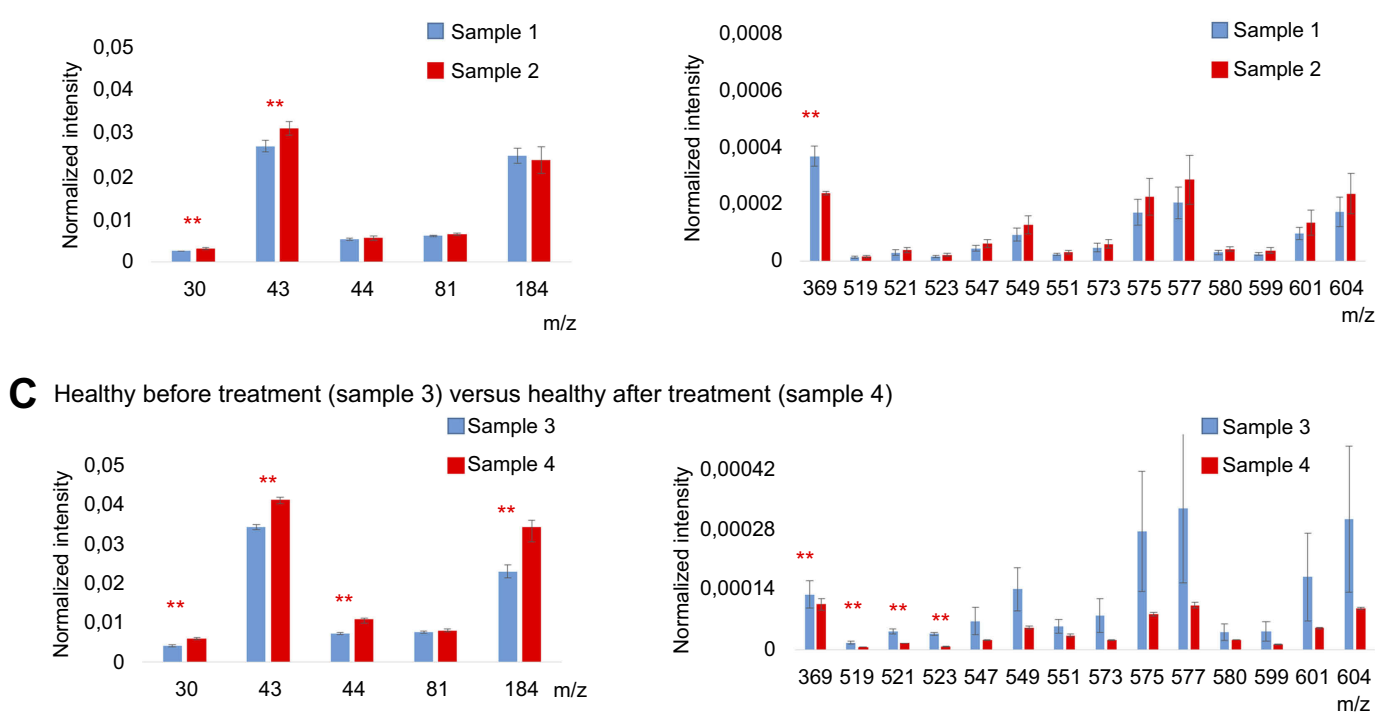

Figure 2 Intensity histograms from time-of-flight secondary ion mass spectrometry measurements on the epidermal region in skin tissue from untreated lesional and healthy and treated lesional and healthy biopsies: Normalized intensity for collagen species at $\mathrm{m} / \mathrm{z} 30,43,44$, and 81 , for phosphatidylcholine head group at $\mathrm{m} / \mathrm{z} \mathrm{I} 84$, for cholesterol at $\mathrm{m} / \mathrm{z} 369$ and diacylglycerol lipid species, in (A) lesional sample I (blue) vs healthy sample 3 (red), (B) lesional sample I (blue) vs NBUVB-treated lesional sample 2 (red), and $(\mathbf{C})$ healthy sample 3 vs NBUVB-treated sample 4 . In all charts, the bar heights show the average value and the error bars show the $95 \% \mathrm{Cl}$ for each peak from three regions of interest from the epidermis. **Significant at $p \leq 0.05$.

Abbreviation: NBUVB, narrowband ultraviolet $B$. 
To visualize and localize these species on tissue sections, they are separately selected and imaged across all samples (Figure S5). The data seen in the statistical assessment are consistent with the distribution in the images, in particular for the healthy treated sample 4 shown in Figure S5 (iv), in which collagen $(\mathrm{m} / \mathrm{z} 30,43,44$, and 81$)$ as well as phosphatidylcholine $(\mathrm{m} / \mathrm{z} 184)$ are highly increased compared to the corresponding image data from the other samples. The cholesterol $(m / z$ 369) signal is clearly reduced in the healthy treated sample 4 .

\section{Distribution of collagen and lipid species in dermis}

The dermis region is the second layer of the skin after the epidermis, and the location is indicated as green lines in the bright-field images of Figure 3. From the CARS channel (red), the lesional sample 1 and the healthy tissue sample 3 have areas of high amount of $\mathrm{C}-\mathrm{H}$ bonds, identified as lipids such as cholesterol and DAGs by ToFSIMS in the dermis area (as shown by white arrows in the CARS channel). After NBUVB treatment, both healthy samples 2 and 4 show that the lipid content appear to become more uniform in the dermis, suggesting a change in the tissue to a healthy state after treatment. In addition, the collagen signal from the SHG channel in the dermis region appears bundled up in the lesional tissue before treatment in sample 1; after treatment with NBUVB in sample 2, the collagen becomes more uniformly distributed. In comparison to the healthy tissue before treatment, sample 3, and after treatment, sample 4, the collagen appears more uniform and composed of small, thin fibers.

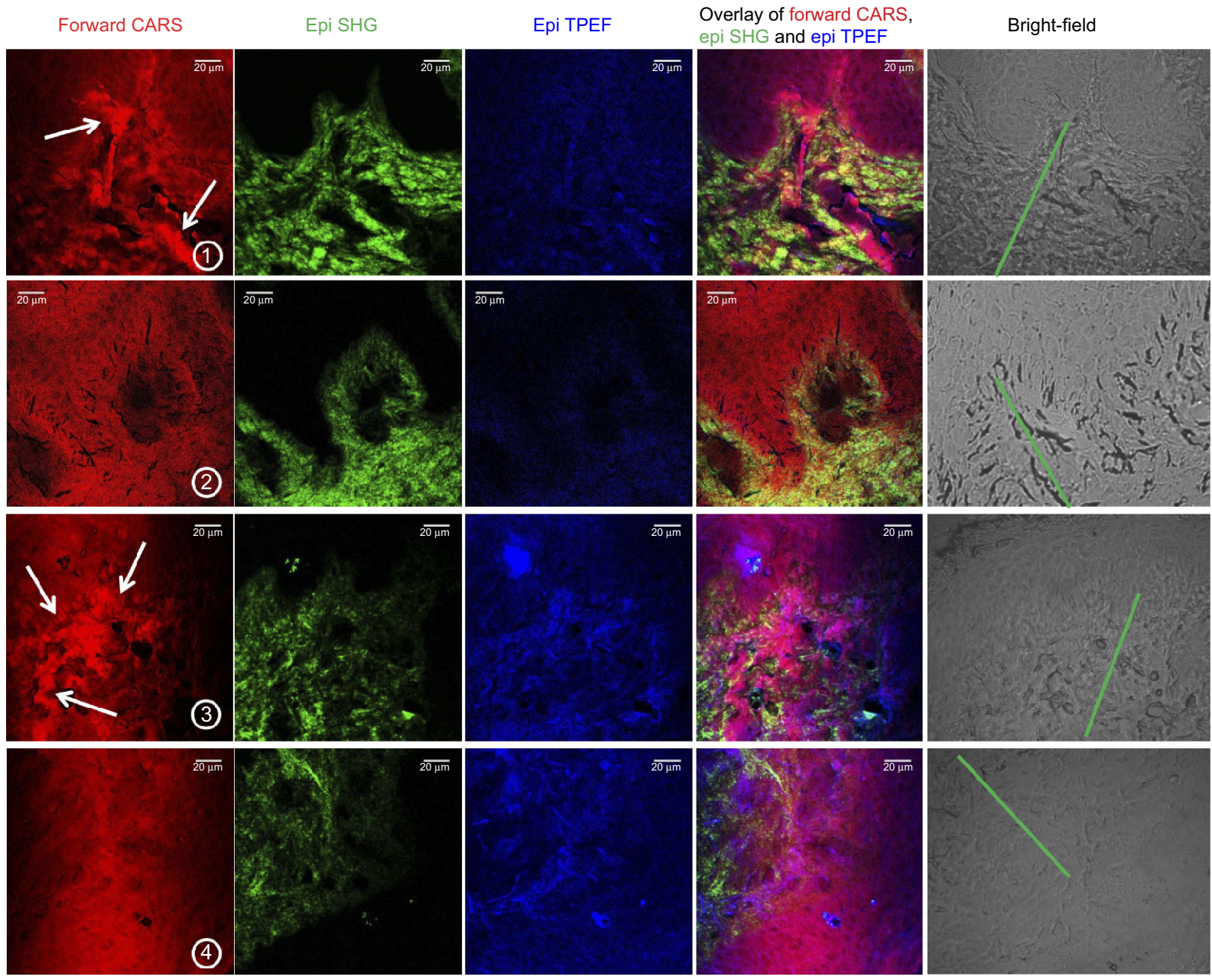

Figure 3 Non-linear microscopy of dermal region of skin tissue for healthy and treated biopsies: display of forward CARS, epi SHG, and epi TPEF, overlay of CARS, SHG, and TPEF, and bright-field images in the dermis region of the skin for each sample condition. Row I: lesional psoriasis tissue before treatment; row 2: psoriasis tissue treated with NBUVB; row 3: healthy tissue; and row 4: healthy tissue treated with NBUVB. The bright-field images indicate the dermis (green line). Scale bar: 20 um. Abbreviations: CARS, coherent anti-Stokes Raman spectroscopy; NBUVB, narrowband ultraviolet B; SHG, second-harmonic generation microscopy; TPEF, two-photon excitation fluorescence microscopy. 
Hence, NBUVB treatment appears to be able to restore the collagen in lesional tissue to a state similar to collagen in healthy tissues, which corresponds well with the fact that NBUVB is known not only to increase collagen levels but also to homogenize collagen in the skin tissue. ${ }^{53,54}$

The TPEF signals decrease for samples 2 and 4, ie, after NBUVB treatment with respect to their respective counterparts, thus indicating the same phenomena observed with collagen but now with elastin. Looking at the overlay of CARS and SHG images of the lesional sample 1, it is clear that collagen and lipids appear in separate regions, not overlapping each other, in the diseased sample 1. In contrast, after treatment, the two components, lipids and collagen, seem to occupy the same region.
MSI was also used in the dermis to track changes in signal intensity of collagen and lipid species in the samples, as shown in Figure 4. Two samples, from lesional tissue before treatment (sample 1) and healthy tissue before treatment (sample 3), were statistically compared, as shown in Figure 4(A). The collagen-related peaks $(\mathrm{m} / \mathrm{z}$ 30,43 , and 44) are noticeably enhanced, whereas cholesterol $(\mathrm{m} / \mathrm{z} 369)$ and some DAG species $(\mathrm{m} / z$ 519, 521, and 549) are substantially decreased in the healthy sample before treatment (sample 3). When comparing the lesional samples before and after treatment (samples 1 and 2, respectively), as illustrated in Figure 4(B), it is revealed that in the NBUVB-treated sample 2, the collagen-related peaks $(\mathrm{m} / \mathrm{z} 30,43$, and 44$)$ are significantly increased and

A Lesional before treatment (sample 1) versus healthy before treatment (sample 3)

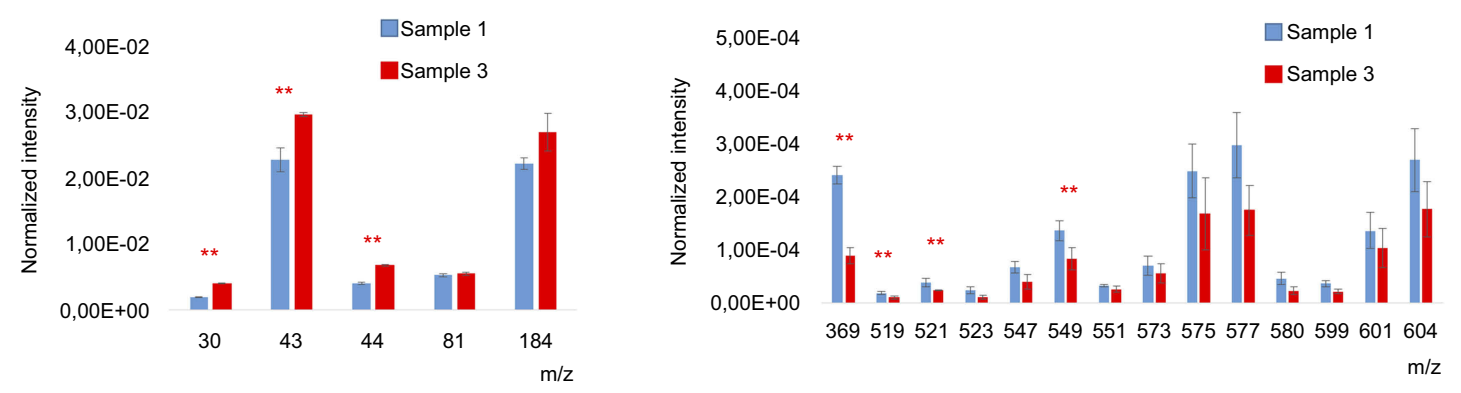

B Lesional before treatment (sample 1) versus lesional after treatment (sample 2)

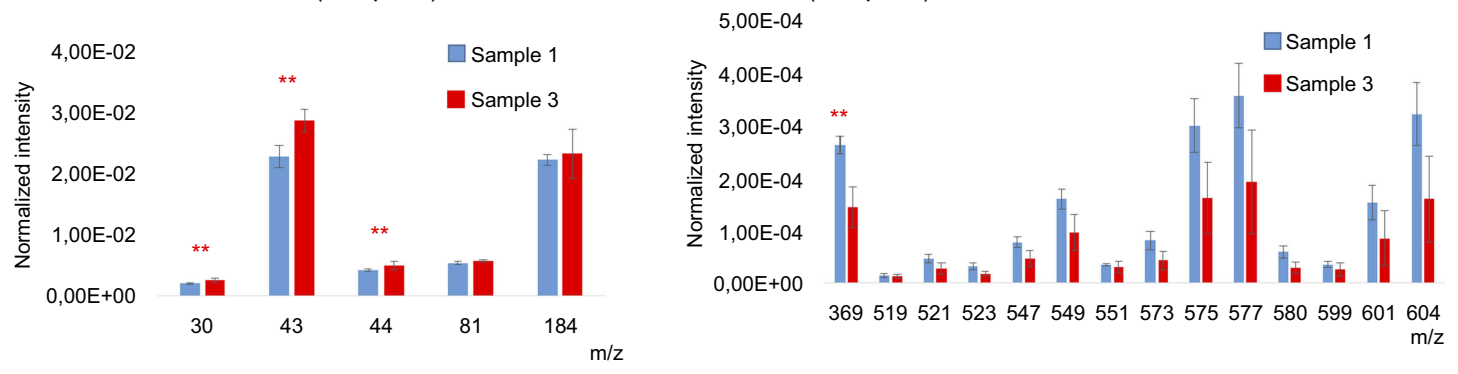

C Healthy before treatment (sample 3) versus healthy after treatment (sample 4)

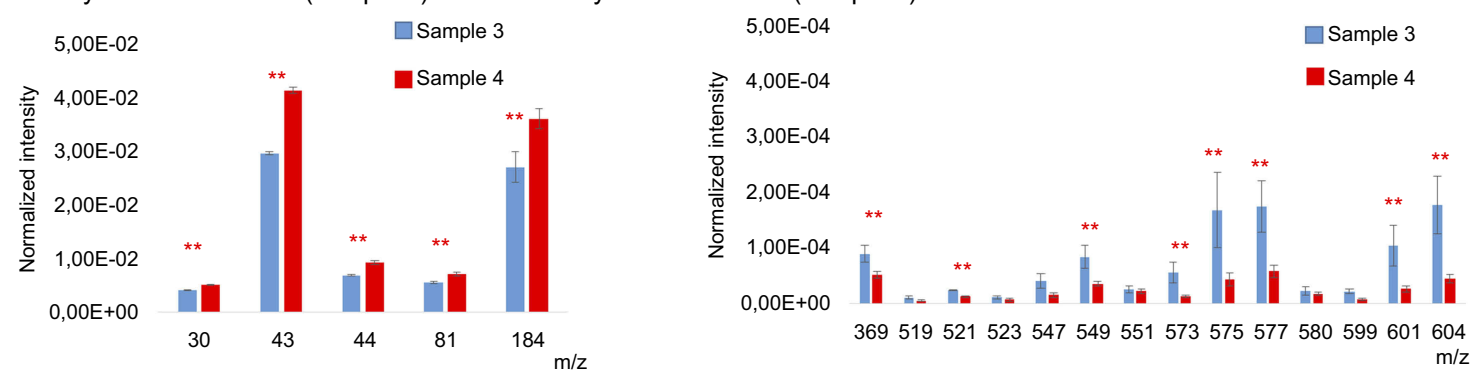

Figure 4 Intensity histograms from time-of-flight secondary ion mass spectrometry measurements on the dermis region in skin tissue from untreated lesional and healthy and NBUVB-treated lesional and healthy biopsies. Normalized intensity for collagen species at $\mathrm{m} / \mathrm{z} 30,43,44$, and 81 , for phosphatidylcholine head group at $\mathrm{m} / \mathrm{z}$ I84, for cholesterol at m/z 369 and diacylglycerol lipid species, in (A) lesional sample I (blue) vs healthy sample 3 (red), (B) lesional sample I (blue) vs NBUVB-treated lesional sample 2 (red), and (C) healthy sample 3 vs NBUVB-treated healthy sample 4 . In all charts, the bar heights show the average value and the error bars show $95 \%$ Cl for each peaks from three regions of interest on the dermis region of each skin tissue section.

Abbreviation: NBUVB, narrowband ultraviolet B. 
the cholesterol $(m / z$ 369) signal is significantly lowered. Comparing the healthy samples 3 and 4, before and after treatment, respectively, as shown in Figure 4(C), indicates a considerable increase for all collagen-related peaks $(\mathrm{m} / \mathrm{z}$ $30,43,44$, and 81$)$ as well as phosphatidylcholine $(\mathrm{m} / \mathrm{z}$ 184) signals after NBUVB treatment, while signals of cholesterol $(m / z$ 369) and also some DAG species $(\mathrm{m} / \mathrm{z}$ $523,573,575,577,602$, and 604) are significantly decreased in the treated sample 4.

Looking at corresponding image data on the dermis region of tissue sections illustrated in Figure S6, it can be clearly seen that collagen signals $(\mathrm{m} / \mathrm{z} 30,43,44$, and 81) have higher intensities in the healthy tissue before treatment (sample 3) than in the lesional tissue before treatment (sample 1) (shown in Figure S6 (iii) and (i), respectively). Also, in the healthy NBUVB-treated sample 4 shown in Figure S6 (iv), collagen $(\mathrm{m} / \mathrm{z} 30,43,44$, and $81)$ and phosphatidylcholine $(\mathrm{m} / z$ 184) signals are noticeably enhanced compared to the corresponding image data from the other samples, whereas cholesterol $(\mathrm{m} / \mathrm{z} 369)$ signals show a significant decrease in the healthy tissue NBUVB-treated sample 4. These observations match well with the corresponding statistical evaluations explained in detail in the previous paragraph.

\section{Discussion}

It is evident from both NLM and ToF-SIMS data in the epidermis region that the lipidic content as observed in the epithelium (from NLM) is primarily composed of cholesterol (from ToF-SIMS) in the lesional tissue before treatment (sample 1) as compared to the healthy tissues (samples 3 and 4). This effect of an increase in cholesterol, especially in blood plasma and epidermis regions of the skin, has already been found in patients with severe psoriasis, who later develop issues related to high cholesterol, or vice versa. ${ }^{55,56}$ After NBUVB treatment on lesional tissue of sample 2, the cholesterol level decreases, as visualized by NLM and ToF-SIMS.

Overall, in both epidermis and dermis regions of skin tissue, collagen is increased after NBUVB treatment. We observed a lower amount of collagen in the lesional samples 1 and 2 compared to the healthy samples 3 and 4 before and after treatment. Collagen was significantly increased in lesional tissue after treatment (sample 2) compared to lesional tissue before treatment (sample 1) after the NBUVB treatment, but this amount was still less than the amount of collagen in sampled 3 and 4, which were considered healthy.
The highest amount of collagen was detected in sample 4, a healthy sample after NBUVB treatment.

In addition, cholesterol and some specific DAG lipid species were significantly enhanced in the lesional samples compared to the healthy samples. No significant changes in DAG components were observed in sample after NBUVB treatment. No significant changes of phosphatidylcholine could be seen, either between the lesional samples 1 and 2 or between the lesional sample 1 and the healthy sample 3. Yet, phosphatidylcholine was significantly increased in the healthy sample after NBUVB treatment compared to the healthy sample 3; thus, the highest amount of phosphatidylcholine was observed in the healthy tissue after NBUVB treatment.

A more comprehensive characterization of the lipids and collagen in lesional and healthy skin of psoriasis patients is desired to understand the role of these molecules in the pathogenesis of the disease. The visualized changes in the skin by label-free, multimodal imaging could be used not only in diagnostics but also as a potential predictor of response to the psoriasis treatment. There is a lack of biomarkers that can be used to predict the treatment response, and therefore this work could contribute toward the further investigation of chemical changes in the skin related to the severity of the disease or to the efficacy of the obtained treatment, in particular when many new biological drugs are on the march. The NLM tool can also be used in vivo for immediate visualization of the skin condition with real-time data and images of treatment.

\section{Conclusions}

The combination of the two techniques presented here provides a plethora of information that could further develop the understanding of psoriasis and the effect of NBUVB treatment as compared to routine histopathological evaluation. A direct comparison of histological data cannot be offered since it does not show the lipids or collagen with H\&E stain. The NLM and MSI indicate that NBUVB treatment lowers the lipid content, especially cholesterol content, in the skin. In both epidermis and dermis regions of skin tissue, collagen is increased after the NBUVB treatment. The highest amount of collagen was detected in sample 4, a healthy sample after NBUVB treatment. NLM detected a structural change in the collagen after the NBUVB treatment (see Supplementary materials for details). Clinically, we observed a visual 
effect of healing of the lesional tissue on the surface after NBUVB treatment, leaving behind postinflammatory hyperpigmentation. There was no visual indication of the healing of sublayers from the observation of surface features.

\section{Acknowledgments}

We are particularly thankful to the research nurses Christina Halldin and Birgitta Stare Merelaid for their excellent assistance. This study was supported by the Bridging project grant from the Centre for Skin Research (SkinResQU), Gothenburg, Sweden, the Wallenberg Foundation, and the Swedish Research Council. NRA would like to thank Prof. Annika Enejder for providing the non-linear microscopy laboratory. The current address for NRA is: Nano-imaging and Spectroscopy Laboratory, Faculty of Science, University of Ontario Institute of Technology, Oshawa, ON, L1G 0C5, Canada.

\section{Author contributions}

NRA, AO, and PM designed the research; NRA, MDP, and MSV performed experiments; NRA performed nonlinear microscopy experiments; MDP performed ToFSIMS experiments; MSV prepared the psoriasis samples for experiments; NN conducted the histological evaluation; NRA, MDP, and PM analyzed the data; and NRA, MDP, AO, and PM wrote the manuscript, with contributions from MSV and NN. All authors contributed to data analysis, drafting and revising the article, gave final approval of the version to be published, and agree to be accountable for all aspects of the work.

\section{Disclosure}

The authors report no conflicts of interest in this work.

\section{References}

1. Boehncke W, Schön MP. Psoriasis. Lancet. 2015;386:983-994. doi:10.1016/S0140-6736(15)61137-0

2. Menter A, Gottlieb A, Feldman SR, et al. Guidelines of care for the management of psoriasis and psoriatic arthritis. J Am Acad Dermatol. 2008;58(5):826-850. doi:10.1016/j.jaad.2008.02.039

3. Icen M, Crowson CS, McEvoy MT, Dann FJ, Gabriel SE, Maradit Kremers H. Trends in incidence of adultonset psoriasis over three decades: a population-based study. J Am Acad Dermatol. 2009;60 (3):394-401. doi:10.1016/j.jaad.2008.10.062

4. Kist JM, Van Voorhees AS. Narrowband ultraviolet B therapy for psoriasis and other skin disorders. Adv Dermatol. 2005;21:235-250.

5. Tami Wong BS, Leon Hsu BA, Wilson Liao MD. Phototherapy in psoriasis: a review of mechanisms of action. J Cutan Med Surg. 2013;17:6-12. doi:10.2310/7750.2012.11124
6. Su YH, Fang JY. Drug delivery and formulations for the topical treatment of psoriasis. Expert Opin Drug Deliv. 2008;5(2):235-249. doi:10.1517/17425247.5.2.235

7. Slominski AT, Zmijewski MA, Plonka PM, Szaflarski JP, Paus R. How UV light touches the brain and endocrine system through skin, and why. Endocrinology. 2018;159(5):1992-2007. doi:10.1210/ en.2017-03230

8. Tobin AM, Veale DJ, Fitzgerald O, et al. Cardiovascular disease and risk factors in patients with psoriasis and psoriatic arthritis. $J$ Rheumatol. 2010;37(7):1386-1394. doi:10.3899/jrheum.090822

9. Fortinskaia ES, Torkhovskaia TI, Sharapova GIA, et al. Features of distribution of free and esterified cholesterol in the epidermis, biological membranes and plasma lipoproteins in psoriasis. Klin Lab Diagn. 1996;4:38-43.

10. Motta S, Monti M, Sesana S, Mellesi L, Ghidoni R, Caputo R. Abnormality of water barrier function in psoriasis. Role of ceramide fractions. Arch Dermatol. 1994;130(4):452-456.

11. Tsambaos D, Kalofoutis A, Stratigos J, Miras C, Capetanakis J. Thinlayer chromatography of phospholipid components of normal and psoriatic epidermis. $B r \quad J$ Dermatol. 1977;97(2):135-138. doi:10.1111/bjd.1977.97.issue-2

12. Ghadially R, Reed JT, Elias PM. Stratum corneum structure and function correlates with phenotype in psoriasis. $J$ Invest Dermatol. 1996;107(4):558-564.

13. Holzer M, Wolf $\mathrm{P}$, Curcic $\mathrm{S}$, et al. Psoriasis alters HDL composition and cholesterol efflux capacity. J Lipid Res. 2012;53(8):1618-1624. doi:10.1194/j1r.M027367

14. Mallbris L, Granath F, Hamsten A, Ståhle M. Psoriasis is associated with lipid abnormalities at the onset of skin disease. $\mathrm{J} \mathrm{Am} \mathrm{Acad}$ Dermatol. 2006;54(4):614-621. doi:10.1016/j.jaad.2005.11.1079

15. Lallas A, Kyrgidis A, Tzellos TG, et al. Accuracy of dermoscopic criteria for the diagnosis of psoriasis, dermatitis, lichen planus and pityriasis rosea. Br J Dermatol. 2012;166(6):1198-1205. doi:10.1111/ j.1365-2133.2012.10868.x

16. Raychaudhuri SK, Maverakis E, Raychaudhuri SP. Diagnosis and classification of psoriasis. Autoimmun Rev. 2014;13(4-5):490-495. doi:10.1016/j.autrev.2014.01.008

17. Heuke S, Vogler N, Meyer T, et al. Multimodal mapping of human skin. Br J Dermatol. 2013;169(4):794-803. doi:10.1111/ bjd. 12427

18. Mazza D, Bianchini P, Caorsi V, et al. Non-linear microscopy. In: Pavesi L, Fauchet PM, editors. Biophotonics. Berlin: Springer; 2008:47-69.

19. Cornett DS, Reyzer ML, Chaurand P, Caprioli RM. MALDI imaging mass spectrometry: molecular snapshots of biochemical systems. Nat Methods. 2007;4:828-833. doi:10.1038/nmeth1094

20. McDonnell LA, Heeren RM. Imaging mass spectrometry. Mass Spectrom Rev. 2007;26:606-643. doi:10.1002/mas.20124

21. Evans CL, Potma EO, Puoris'haag M, et al. Chemical imaging of tissue in vivo with video-rate coherent anti-Stokes Raman scattering microscopy. Proc Natl Acad Sci U S A. 2005;102(46):16807-16812. doi:10.1073/pnas.0508282102

22. Meyer T, Bergner N, Bielecki C, et al. Nonlinear microscopy, infrared, and Raman microspectroscopy for brain tumor analysis. $J$ Biomed Opt. 2011;16(2):21110-21113. doi:10.1117/1.3533268

23. Shuhua Y, Slipchenko MN, Cheng JX. Multimodal nonlinear optical microscopy. Laser Photon Rev. 2011;5(4):496-512. doi:10.1002/ lpor.201000027

24. Lei M, Winterhalder M, Selm R, et al. Video-rate wide-field coherent anti-Stokes Raman scattering microscopy with collinear nonphasematching illumination. J Biomed Opt. 2011;16(2):21102. doi:10.1117/1.3533707

25. Evans CL, Xie XS. Coherent anti-stokes Raman scattering microscopy: chemical imaging for biology and medicine. Annu Rev Anal Chem (Palo Alto Calif). 2008;1:883-909. doi:10.1146/annurev. anchem.1.031207.112754 
26. Pezacki JP, Blake JA, Danielson DC, Kennedy DC, Lyn RK, Singaravelu R. Chemical contrast for imaging living systems: molecular vibrations drive CARS microscopy. Nat Chem Biol. 2011;7 (3):137-145. doi:10.1038/nchembio. 525

27. Pena A, Strupler M, Boulesteix T, Schanne-Klein M. Spectroscopic analysis of keratin endogenous signal for skin multiphoton microscopy. Opt Express. 2005;13(16):6268-6274.

28. Laiho LH, Pelet S, Hancewicz TM, Kaplan PD, So PTC. Two-photon 3-D mapping of ex vivo human skin endogenous fluorescence species based on fluorescence emission spectra. J Biomed Opt. 2005;10 (2):024016. doi:10.1117/1.1891370

29. Nakamura O. Fundamental of two-photon microscopy. Microsc Res Tech. 1999;47(3):165-171. doi:10.1002/(ISSN)1097-0029

30. Cicchi R, Vogler N, Kapsokalyvas D, Dietzek B, Popp J, Pavone FS. From molecular structure to tissue architecture: collagen organization probed by SHG microscopy. J Biophotonics. 2013;6(2):129-142. doi:10.1002/jbio.v6.2

31. Konig K, Riemann I. High-resolution multiphoton tomography of human skin with subcellular spatial resolution and picosecond time resolution. J Biomed Opt. 2003;8(3):432-439. doi:10.1117/1.1577349

32. Malmberg P, Jennische E, Nilsson D, et al. High-resolution, imaging TOF-SIMS: novel applications in medical research. Anal Bioanal Chem. 2011;399(8):2711-2718. doi:10.1007/s00216-010-3679-7

33. Passarelli MK, Winograd N. Lipid imaging with time-of-flight secondary ion mass spectrometry (ToF-SIMS). Biochim Biophys Acta. 2011;1811(11):976-990. doi:10.1016/j.bbalip.2011.05.007

34. Brunelle A, Touboul D, Laprévote O. Biological tissue imaging with time-of-flight secondary ion mass spectrometry and cluster ion sources. J Mass Spectrom. 2005;40(8):985-999. doi:10.1002/(ISSN)1096-9888

35. Brunelle A, Laprévote O. Lipid imaging with cluster time-of-flight secondary ion mass spectrometry. Anal Bioanal Chem. 2009;393:3135. doi:10.1007/s00216-008-2384-2

36. Malmberg P, Börner K, Chen Y, et al. Localization of lipids in the aortic wall with imaging TOF-SIMS. Biochim Biophys Acta. 2007;1771(2):185-195. doi:10.1016/j.bbalip.2006.12.003

37. Malmberg P, Karlsson T, Svensson H, et al. A new approach to measuring vitamin $\mathrm{D}$ in human adipose tissue using time-of-flight secondary ion mass spectrometry: a pilot study. J Photochem Photobiol B Biol. 2014;138:295-301. doi:10.1016/j.jphotobiol.2014.06.008

38. Nygren H, Börner K, Malmberg P, Tallarek E, Hagenhoff B. Imaging TOF-SIMS of rat kidney prepared by high-pressure freezing. Microsc Res Tech. 2005;68(6):329-334. doi:10.1002/(ISSN)1097-0029

39. Sjövall P, Lausmaa J, Johansson B. Mass spectrometric imaging of lipids in brain tissue. Anal Chem. 2004;76:4271-4278. doi:10.1021/ ac049389p

40. Breunig HG, Bückle R, Kellner-Höfer M, et al. Combined in vivo multiphoton and CARS imaging of healthy and disease-affected human skin. Microsc Res Tech. 2012;75(4):492-498. doi:10.1002/ jemt.21082
41. Campagnola PJ, Millard AC, Terasaki M, Hoppe PE, Malone CJ, Mohler WA. Three-dimensional high-resolution second-harmonic generation imaging of endogenous structural proteins in biological tissues. Biophys J. 2002;82(1):493-508. doi:10.1016/S0006-3495(02)75414-3

42. Masters BR, So PT, Gratton E. Multiphoton excitation fluorescence microscopy and spectroscopy of in vivo human skin. Biophys J. 1997;72(6):2405-2412. doi:10.1016/S0006-3495(97)78886-6

43. Ståhle M. Psoriasis: in between the skin and the fat. Exp Dermatol. 2015;24(3):181-182. doi:10.1111/exd.2015.24.issue-3

44. Fitzpatrick TB. The validity and practicality of sun-reactive skin types I through VI. Arch Dermatol. 1988;124:869-871. doi:10.1001/archderm.1988.01670060015008

45. Sodhi RNS. Time-of-flight secondary ion mass spectrometry (TOFSIMS): versatility in chemical and imaging surface analysis. Analyst. 2004;129:483-487. doi:10.1039/b402607c

46. He C, Hu X, Jung RS, et al. High-resolution imaging and quantification of plasma membrane cholesterol by NanoSIMS. PNAS. 2017;114 (8):201621432.

47. Pour MD, Jennische E, Lange S, Ewing AG, Malmberg P. Foodinduced changes of lipids in rat neuronal tissue visualized by ToFSIMS imaging. Scientific Rep. 2016;6:32797. doi:10.1038/srep32797

48. Ahad NA, Yahaya SSS. Sensitivity analysis of Welch's t-test. AIP Conf Proc. 2014;1605:888.

49. Deniset-Besseau A, Duboisset J, Benichou E, Hache F, Brevet PF, Schanne-Klein MC. Measurement of the second-order hyperpolarizability of the collagen triple helix and determination of its physical origin. $J$ Phys Chem B. 2009;113:13437-13445. doi:10.1021/jp9046837

50. Elias PM. Epidermal lipids, membranes and keratinization. Int $J$ Dermatol. 1981;20:1-19.

51. Ishida-Yamamoto A, Simon M, Kishibe M, et al. Epidermal lamellar granules transport different cargoes as distinct aggregates. J Invest Dermatol. 2004;122(5):1137-1144. doi:10.1111/j.0022-202X.2004. 22515.x

52. Weinigel M, Breunig HG, Darvin ME, et al. Impact of refractive index mismatches on coherent anti-Stokes Raman scattering and multiphoton autofluorescence tomography of human skin in vivo. Phys Med Biol. 2015;60(17):6881-6899. doi:10.1088/0031-9155/60/17/6881

53. Boyer B, Fourtanier A, Kern P, Labat-Robert J. UVA- and UVBinduced changes in collagen and fibronectin biosynthesis in the skin of hairless mice. J Photochem Photobiol B Biol. 1992;14(3):247-259. doi:10.1016/1011-1344(92)85102-Z

54. Trautinger F, Mazzucco K, Knobler RM, Brenner RE, Zabel B, Müller PK. UVA- and UVB-induced changes in hairless mouse skin collagen. Arch Dermatol Res. 1994;286(8):490-494.

55. Feingold KR, Grunfeld C. Psoriasis: it's more than just the skin. $J$ Lipid Res. 2012;53(8):1427-1429. doi:10.1194/jlr.E029330

56. Pietrzak A, Michalak-Stoma A, Chodorowska G, Szepietowski JC. Lipid disturbances in psoriasis: an update. Mediators Inflamm. 2010;2010:1-12. doi:10.1155/2010/535612 


\section{Supplementary material}
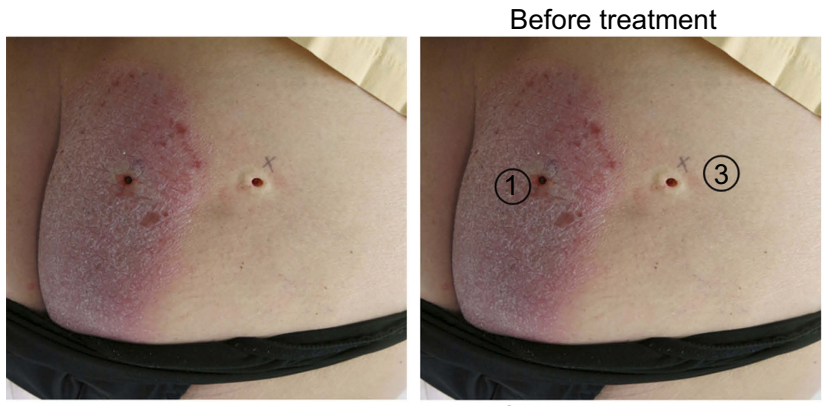

After treatment

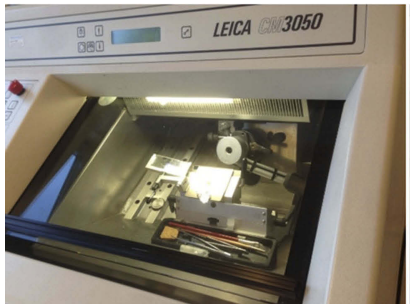

Sample preparation

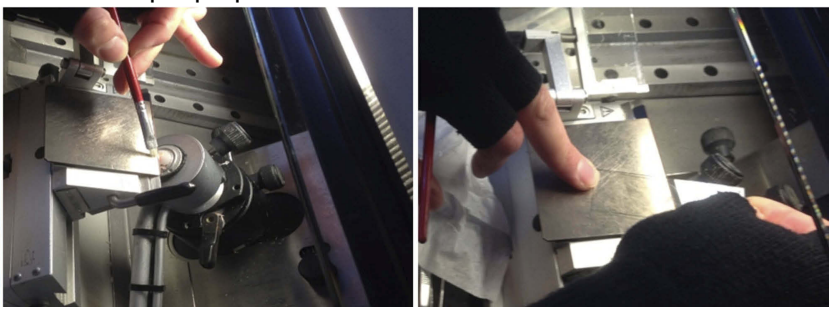

Figure SI Row I: Images depicting the psoriatic lesion of the patient with regions from where biopsies were obtained, with numbering of samples. Row 2: pictures illustrating the sample preparation method with cryo-sectioning instrument.
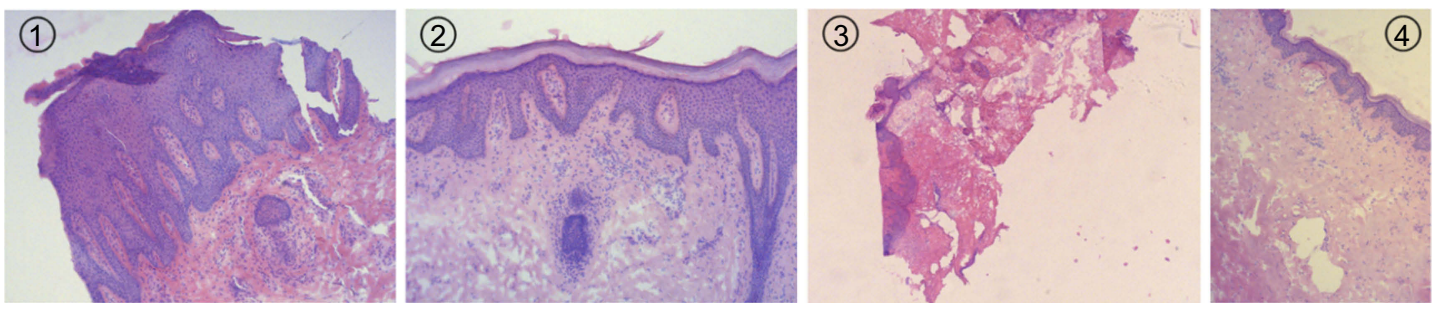

Figure S2 Histopathological images from H\&E staining of skin tissue from lesional healthy and narrowband ultraviolet B (NBUVB)-treated biopsies. Optical microscopy images of freeze-dried skin tissue sections in lesional psoriasis tissue before treatment (sample I), NBUVB-treated lesional tissue (sample 2), healthy tissue (sample 3), and NBUVBtreated lesional tissue (sample 4). 


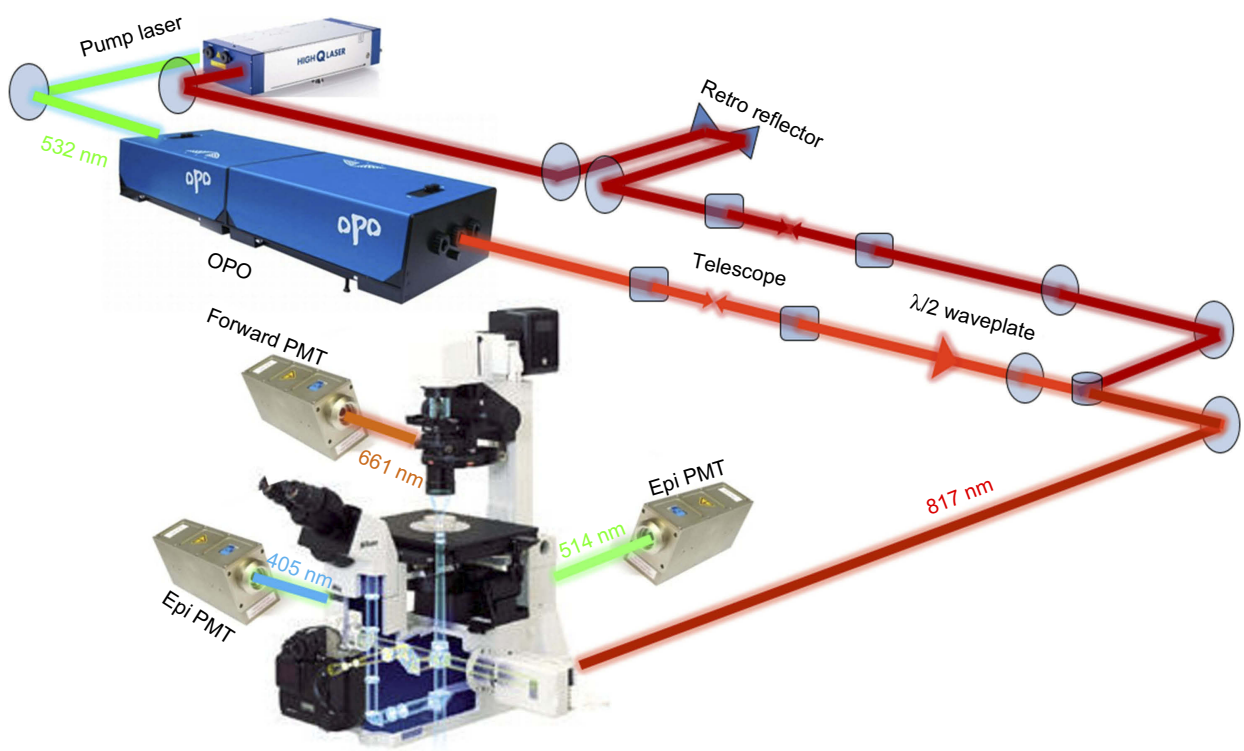

Figure S3 Non-linear microscopy set-up diagram showing collection of coherent anti-Stokes Raman spectroscopy (CARS) (66I nm), two-photon excitation fluorescence (TPEF) $(5 / 4 \mathrm{~nm})$, and second-harmonic generation (SHG) $(405 \mathrm{~nm})$ signals with incoming beams of 1064 and $817 \mathrm{~nm}$.

A
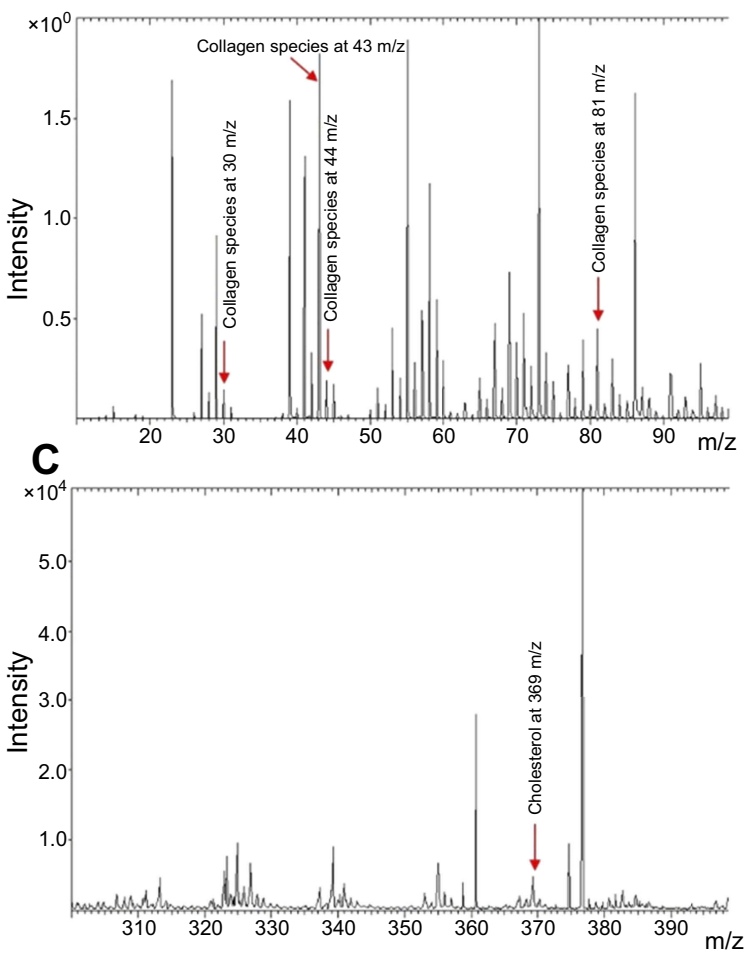

\section{B}
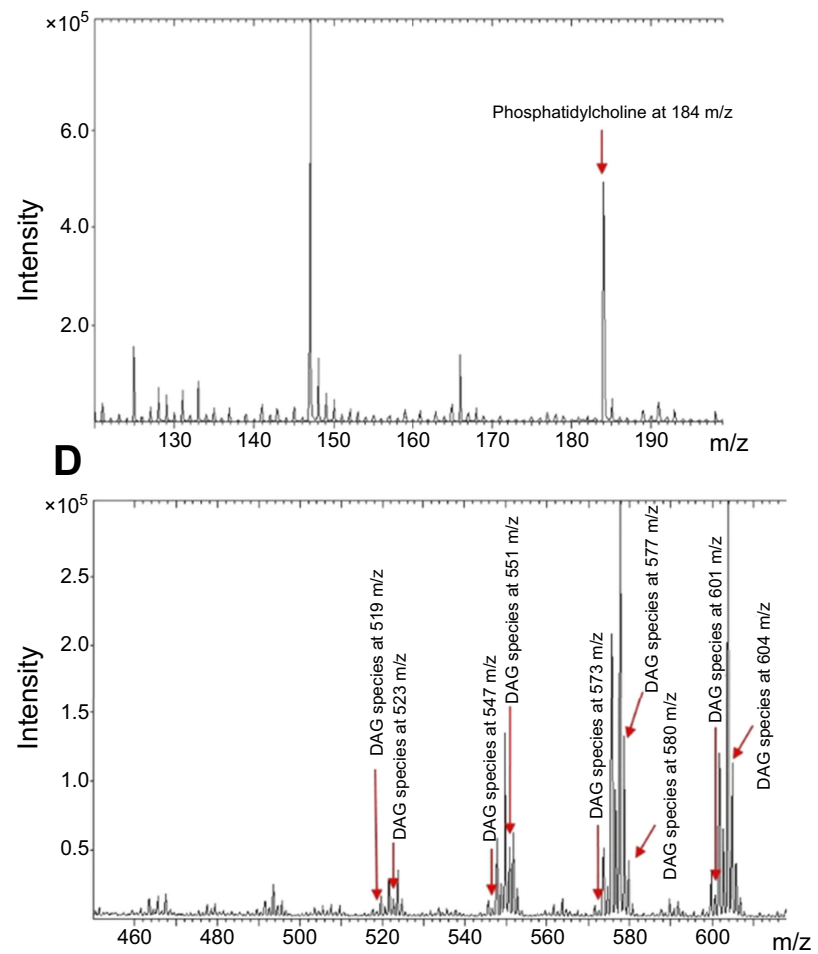

Figure S4 Representative time-of-flight secondary ion mass spectrometry mass spectrum from a tissue section including (A) collagen species at $m / z$ 30, $m / z$ 43, $m / z 44$, and $\mathrm{m} / \mathrm{z}$ 8I; (B) phosphatidylcholine head group at $\mathrm{m} / \mathrm{z} \mathrm{I84;} \mathrm{(C)} \mathrm{cholesterol} \mathrm{at} \mathrm{m} / \mathrm{z} 369$; and (D) diacylglycerol (DAG) lipid species at $\mathrm{m} / \mathrm{z} 5 \mathrm{I}, \mathrm{m} / \mathrm{z} 523, \mathrm{~m} / \mathrm{z} 547, \mathrm{~m} / \mathrm{z} 55 \mathrm{I}, \mathrm{m} / \mathrm{z}$ $573, \mathrm{~m} / \mathrm{z} 577, \mathrm{~m} / \mathrm{z} 580, \mathrm{~m} / \mathrm{z} 601$, and $\mathrm{m} / \mathrm{z} 604$. 
(i)

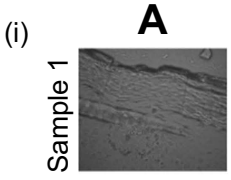

(ii)

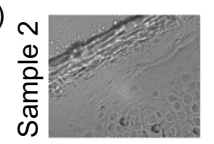

(iii) $m$

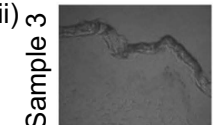

(iv) $\checkmark$

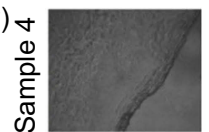

B 30 u
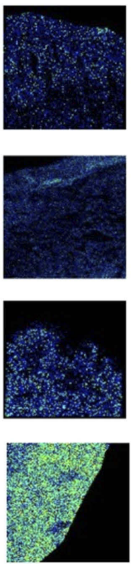
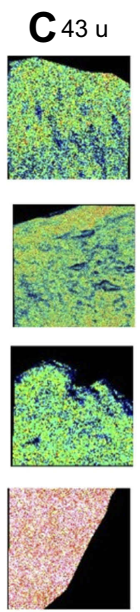
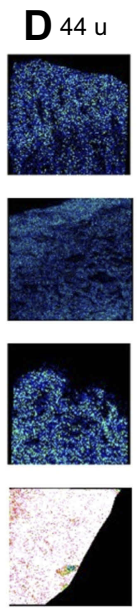
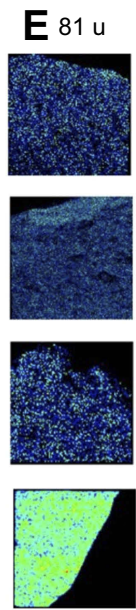

F $184 \mathrm{u}$
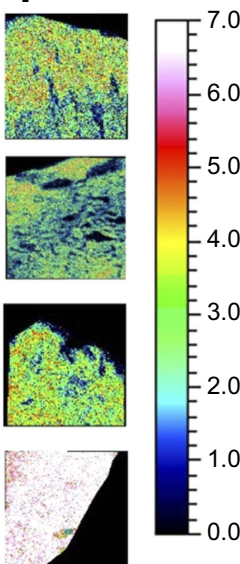

G 369 u

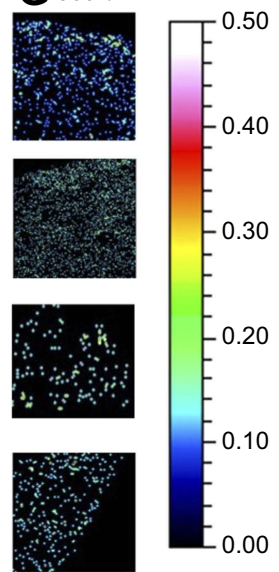

Figure S5 Time-of-flight secondary ion mass spectrometry ion images from the epidermal region of skin tissue from lesional healthy and narrowband ultraviolet B (NBUVB)-treated biopsies. (A) Optical microscopy images of epidermis regions from freeze-dried skin tissue sections in (i) lesional psoriasis tissue before treatment (sample I), (ii) NBUVB-treated lesional tissue (sample 2), (iii) healthy tissue (sample 3), and (iv) NBUVB-treated lesional tissue (sample 4). Specific ion images of (B) $\mathrm{m} / \mathrm{z} 30$, (C) $\mathrm{m} / \mathrm{z}$ 43, (D) $\mathrm{m} / \mathrm{z} 44$, (E) $\mathrm{m} / \mathrm{z} 8 \mathrm{I}$, (F) $\mathrm{m} / \mathrm{z} 184$, and (G) $\mathrm{m} / \mathrm{z} 369$ are shown across all samples. All individual ion images are in a $500 \mu \mathrm{m} \times 500 \mu \mathrm{m}$ field of view and acquired at a pixel resolution of approximately $2 \mu \mathrm{m}$. Images are shown in the color scales provided to the right of the images.

(i)

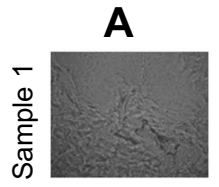

(ii)

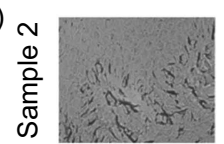

(iii)

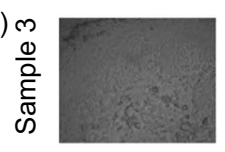

(iv)

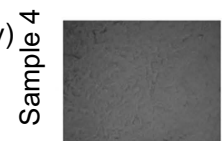

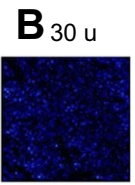
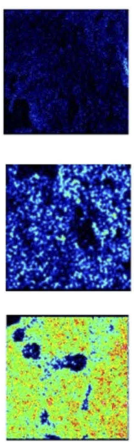
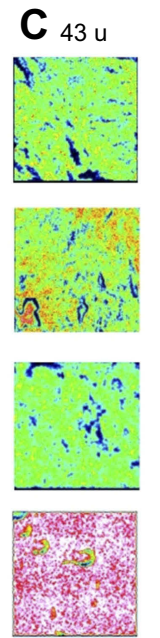
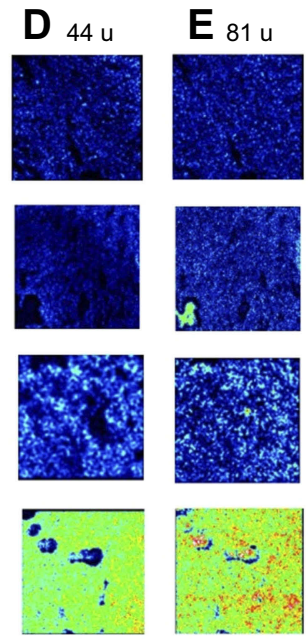
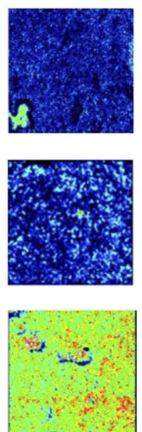
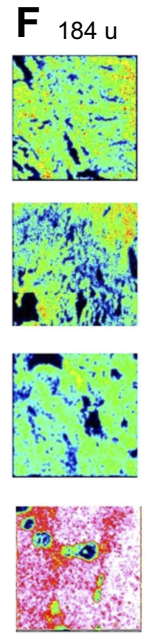
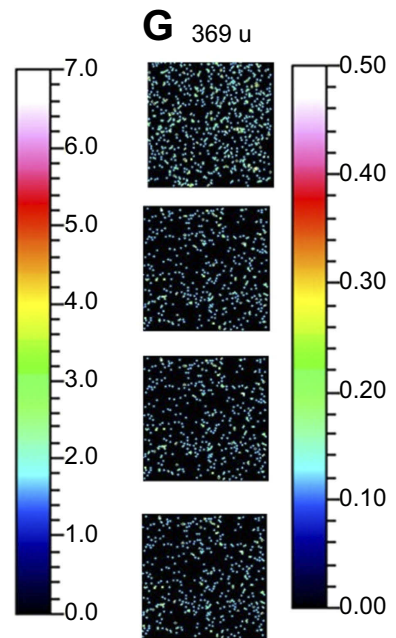

Figure S6 Time-of-flight secondary ion mass spectroscopy ion images from the dermis region of skin tissue from lesional healthy and treated biopsies. (A) Optical microscopy images of epidermis regions from freeze-dried skin tissue sections in (i) lesional psoriasis tissue before treatment (sample I), (ii) narrowband ultraviolet $B$ (NBUVB)-treated lesional tissue (sample 2), (iii) healthy tissue (sample 3), and (iv) NBUVB-treated lesional tissue (sample 4). Specific ion images of (B) $\mathrm{m} / \mathrm{z} 30$, (C) $\mathrm{m} / \mathrm{z} 43$, (D) $\mathrm{m} / \mathrm{z} \mathrm{44,} \mathrm{(E)} \mathrm{m} / \mathrm{z} 8 \mathrm{I}$, (F) $\mathrm{m} / \mathrm{z}$ I84, and (G) $\mathrm{m} / \mathrm{z} 369$ are shown across all samples. All individual ion images are in a $500 \mu \mathrm{m} \times 500 \mu \mathrm{m}$ field of view and acquired at a pixel resolution of approximately $2 \mu \mathrm{m}$. Images are shown in the color scale provided to the right of the images.

Table SI Report of four sample tissues obtained from the patient with chronic plaque psoriasis

\begin{tabular}{|l|l|l|l|}
\hline Sample & Lesional/healthy skin & Before/after NBUVB & Date (mm/dd/yyyy) \\
\hline $\mathrm{I}$ & Lesional & Before & $09 / 25 / 2014$ \\
2 & Lesional & After & $11 / 26 / 2014$ \\
3 & Healthy & Before & $09 / 25 / 2014$ \\
4 & Healthy & After & $11 / 26 / 2014$ \\
\hline
\end{tabular}

Abbreviation: NBUVB, narrowband ultraviolet $B$. 


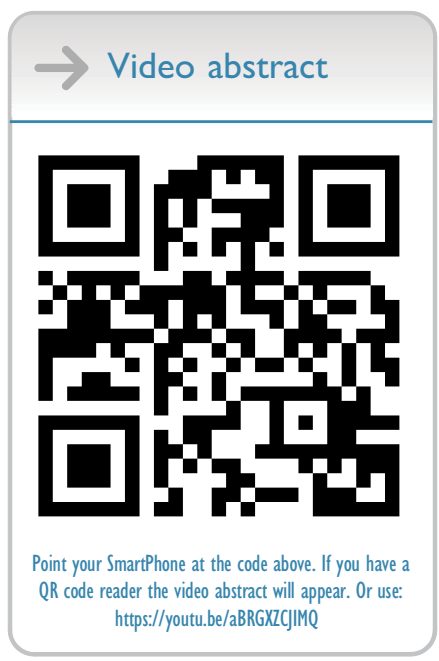

Psoriasis: Targets and Therapy

\section{Publish your work in this journal}

Psoriasis: Targets and Therapy is international, peer-reviewed, open access journal focusing on psoriasis, nail psoriasis, psoriatic arthritis and related conditions, identification of therapeutic targets and the

optimal use of integrated treatment interventions to achieve improved outcomes and quality of life. Visit http://www.dovepress. com/testimonials.php to read real quotes from published authors. 